\title{
Measurements on scaled models of urban environments and comparisons with ray-tracing propagation simulation.
}

\author{
Danilo Erricolo, Member, IEEE, Giuseppe D'Elia, Student Member, IEEE, Piergiorgio L.E. Uslenghi, \\ Fellow, IEEE
}

\begin{abstract}
Scaled models of simple two-dimensional urban environments are considered in order to investigate propagation along a vertical plane. Specifically, path loss measurements are taken for different positions of the transmitting and receiving antennas at $25 \mathrm{GHz}$. Then, measurement results are compared with theoretical predictions computed by a ray-tracing polygonal line simulator. The measurements indicate a very good agreement between the ray-tracing model and the experiments.
\end{abstract}

Keywords - Ray tracing, Radio Propagation, Geometrical Theory of Diffraction, Urban Propagation, Measurements.

\section{INTRODUCTION}

$\mathrm{R}^{\mathrm{A}}$ AY-TRACING methods are usually applied to make path-loss estimates in complex environments such as those found inside cities. In fact, cities are geometrically and electrically too complex to be analyzed using full wave methods. Even with ray-tracing methods, a full threedimensional investigation is very difficult to achieve and, therefore, a simpler two-dimensional problem is examined here. The two-dimensional problem assumes that propagation occurs above building obstructions and that the main contributions come from trajectories contained in a vertical plane passing through the transmitting and receiving antennas. The theoretical path-loss estimates are made using a polygonal line simulator that is based on a ray-tracing method described in [1], [2], [3]. The purpose of this work is the measurement of the accuracy that can be achieved using the polygonal line simulator. In general, the accuracy of a propagation prediction method is of strong interest in the field of wireless communications in order to properly characterize the communication channel. However, the rigorous application of ray-tracing methods requires the computation of many trajectories even for simple geometries. Therefore, in the polygonal line simulator, some approximations were introduced to reduce the number of trajectories. The advantage of the approach considered herein is that the measurements are made in a controlled environment where the assumptions of the polygonal line simulator are satisfied as much as possible. In fact, the measurements are conducted inside an anechoic chamber, on scaled models of simple urban environments so that all parameters of the problem are known. This approach does not intend to

The authors are with the Department of Electrical and Computer Engineering, University of Illinois at Chicago, 1020 SEO (M/C 154), 851 South Morgan St., Chicago, IL, 60607-7053, USA email: derricol@ece.uic.edu. This research was supported by the National Science Foundation under Grant ECS-9979413.

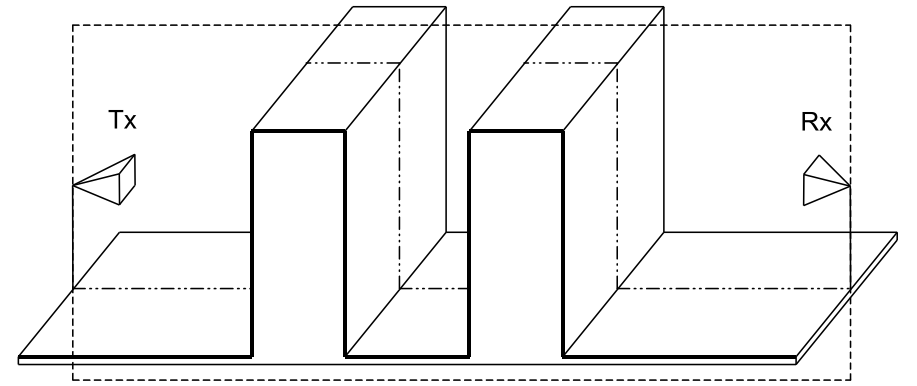

Fig. 1. Example of a scaled model and of the vertical plane along which the trajectories are considered for the theoretical model.

replace actual field measurements, but rather it is meant to provide detailed feedback about the method. On the other hand, detailed feedback cannot be provided by field measurements where too many factors are unknown, it is not possible to relate discrepancies to the method, and the standard deviation of the difference between prediction and measurements is usually above $6 \mathrm{~dB}$.

The paper is organized as follows. A brief description of the polygonal line simulator is given to justify the experimental setup. In particular, the choice of antennas with an appropriate directivity pattern is clarified. Then, the experiments are discussed and comparisons with the theoretical predictions are thoroughly described.

\section{The Polygonal Line Simulator}

The polygonal line simulator is a computer-based prediction tool for two-dimensional wave propagation in urban environments. Wave propagation is considered along the vertical plane joining the transmitting antenna to the receiving antenna, as shown in Fig 1. A detailed description of the simulator is given in [1], [2], [3].

For a given urban environment, the intersection between the vertical plane and the environment is approximated with a polygonal line. With a polygonal line, different building shapes as well as terrain variations are easily taken into account. The simulator computes all the trajectories that lie in the vertical plane, associates with each trajectory an attenuation value, and determines the overall attenuation of the received electric field. The evaluation of the attenuation for each ray path is performed using the Uniform Theory of Diffraction (UTD) [4]. In particular, up to second order UTD diffraction coefficients are employed [5], $[6],[7],[8]$. This avoids field discontinuities that may ap- 


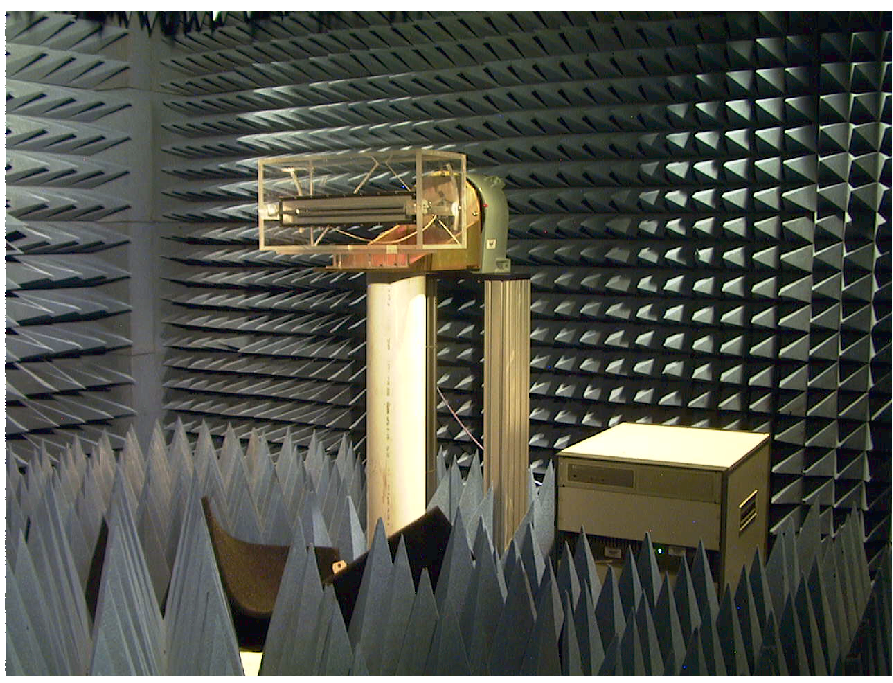

Fig. 2. View of the anechoic chamber at the University of Illinois at Chicago. This facility was installed by Orbit FR and is certified for measurements from $2 \mathrm{GHz}$ to $26.5 \mathrm{GHz}$

pear when one of the antennas crosses a transition region. The model also introduces impedance surfaces in order to electrically characterize each segment of the profile. In addition, the algorithm considers both vertical and horizontal polarizations, and accounts for the directivity of the antennas. A comparison of the polygonal line simulator with the knife-edge method has been addressed in Section $\mathrm{V}$ of [1], whereas comparisons with other methods as well as measurements are discussed in Section VII of [1].

\section{The Experimental Setup}

The experiments have the purpose to verify the accuracy of the polygonal line simulator. The polygonal line simulator is based on a two-dimensional assumption and, therefore, the experiments must approximate, as closely as possible, this assumption. First of all, in order to reduce the influence of external factors, the measurements are taken inside the anechoic chamber facility at the University of Illinois at Chicago, shown in Fig. 2.

The experiments consist of the measurement of the propagation path-loss due to the presence of scaled models of buildings between the transmitting and receiving antennas. In particular, the trajectories considered by the polygonal line simulator are contained in a vertical plane. As a consequence, in order to experimentally emphasize only those trajectories that are contained in the vertical plane, such as trajectory $T_{X} \rightarrow P \rightarrow Q \rightarrow R_{X}$ of Fig. 3, the patterns of both antennas in the horizontal plane must be narrow and directed along the line connecting the antennas.

This directive pattern in the horizontal plane has also an additional reason. Even though, in the theoretical model, the buildings are infinitely large in the transverse direction, the actual scaled models must be truncated at some point. The termination introduces a disturbing contribution by allowing trajectories to propagate around buildings, such as for the trajectory $T_{x} \rightarrow D_{1} \rightarrow D_{2} \rightarrow R_{x}$ of Fig. 3 . Hence, the additional advantage of having a strong direc-

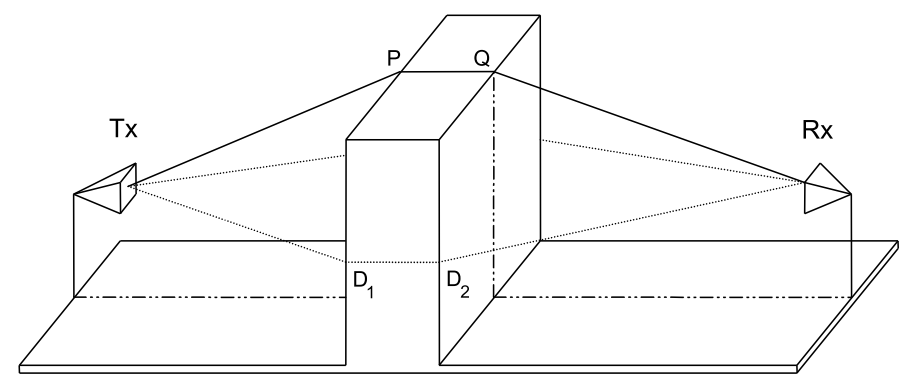

Fig. 3. Example of three-dimensional propagation. The trajectory $T_{x} \rightarrow P \rightarrow Q \rightarrow R_{x}$ that is contained in the vertical plane is of interest for the experiments; however, the trajectory $T_{x} \rightarrow D_{1} \rightarrow$ $D_{2} \rightarrow R_{x}$ is an undesired one.

tivity in the horizontal plane is the capability of reducing the contribution of undesired trajectories that propagate around the scaled buildings.

Furthermore, for both antennas the directivity patterns in the vertical plane must be isotropic, so that the contributions from all possible trajectories are equally weighted. At least within the angular sector of interest shown in Fig. 4, the directivity gain must be reasonably constant. These requirements in the horizontal and vertical planes were met by two high gain sector antennas BCAH90-250 operated at $25 \mathrm{GHz}$ and provided by Andrew Corporation, Orland Park, IL, USA. The directivity patterns for the BCAH90-250 antennas are shown in Fig. 5 and 6 for azimuth and elevation patterns, respectively. The calibration of the BCAH90-250 antennas was performed in free space conditions using standard gain horns operated in the bandwidth from $18 \mathrm{GHz}$ to $26.5 \mathrm{GHz}$. The experiments measure the field $E\left(R_{x}\right)$ that reaches $R_{x}$ after propagating past the scaled building models. During the experiments, $T_{x}$ is fixed while $R_{x}$ is manually moved vertically. Measurement data are plotted in terms of the normalized field $E_{0}\left(R_{x}\right)$, i.e the measured field $E\left(R_{x}\right)$ divided by the magnitude of the field measured for the same position of the antennas in free space $E_{\text {free space }}\left(R_{x}\right)$ :

$$
E_{0}\left(R_{x}\right)=\frac{E\left(R_{x}\right)}{\left|E_{\text {free space }}\left(R_{x}\right)\right|} .
$$

Two polarizations are considered: hard and soft. Hard polarization corresponds to a Neumann boundary condition, which occurs when the incident electric field is perpendicular to the diffracting edge, and is also known as vertical polarization. Soft polarization corresponds to a Dirichlet boundary condition, which occurs when the incident electric field is parallel to the diffracting edge, and is also known as horizontal polarization.

The scaled models are made of copper to obtain a perfect electric conductor behavior that strengthens the effects of reflections. The geometrical size of the models is determined mainly by the dimension of the anechoic chamber; within these limits, their width was chosen to be as large as possible to reduce the contributions from undesired trajectories propagating around the scaled models. Moreover, absorbing material was wrapped around corners and edges to further reduce unwanted contributions. 


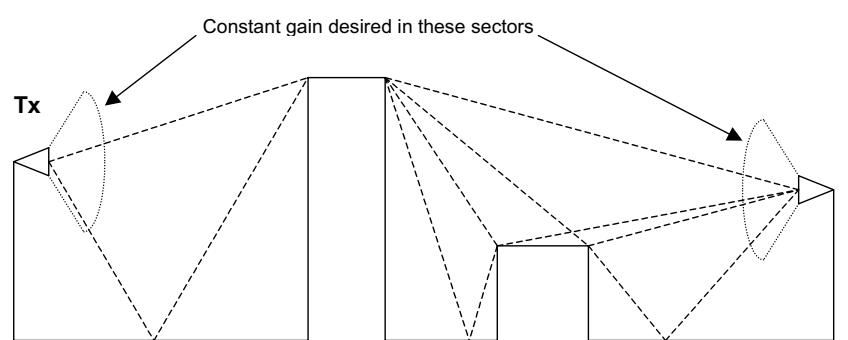

Fig. 4. Example of the angular sectors within which the directive gain must be as constant as possible to guarantee that all trajectories of interest are equally weighted.

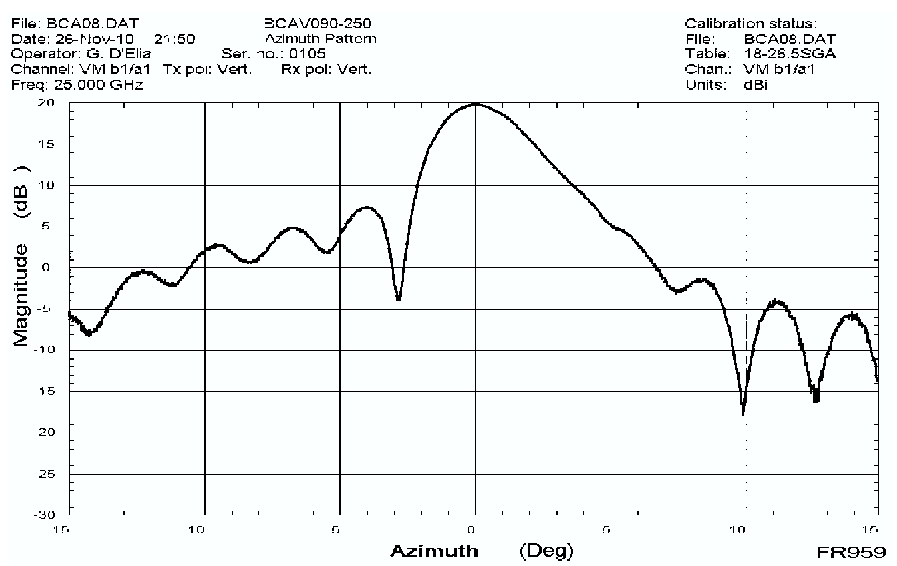

Fig. 5. Azimuth pattern for the Andrew BCAH090-250 high gain sector antenna. The strong directivity ensures that any undesired trajectory, such as $T_{x} \rightarrow D_{1} \rightarrow D_{2} \rightarrow R_{x}$ of Fig. 3 , is weighted at least $20 \mathrm{~dB}$ less than the desired trajectories that lie in the vertical plane.

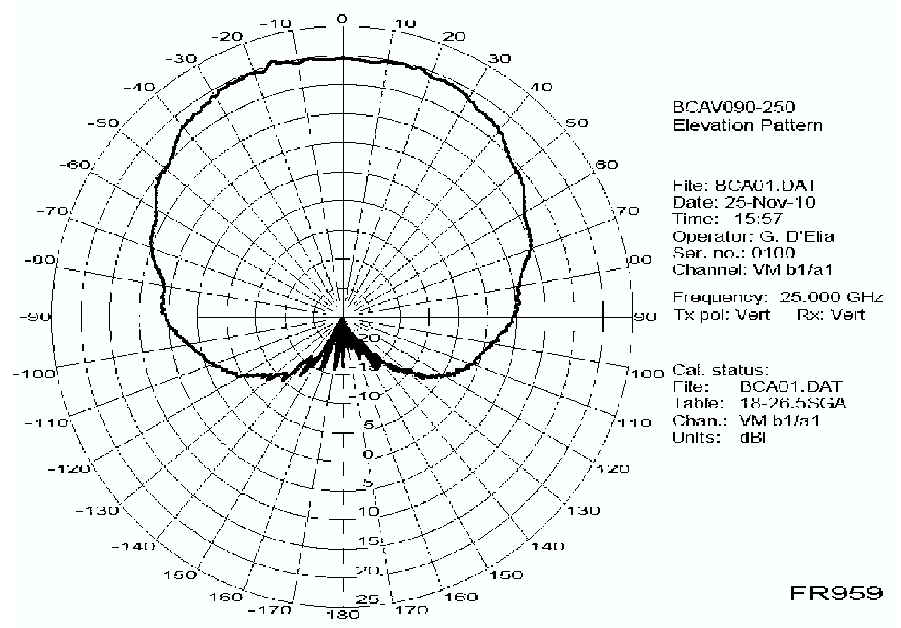

Fig. 6. Elevation pattern for the Andrew BCAH090-250 high gain sector antenna. The gain is within $1 \mathrm{~dB}$ of the maximum value inside an angle of $60^{\circ}$ and within $3 \mathrm{~dB}$ of the maximum value inside an angle of $80^{\circ}$. This pattern satisfies the requirements explained in Fig. 4.
The geometrical shapes of the scaled models are simple but effective to test the performance of the polygonal line simulator. The following shapes are examined:

- Single-building profile. This shape represents a singlebuilding obstruction, as shown in Fig. 3 and it is used because a rectangular shape approximates a building more accurately then the previously used knife-edge approximation, as pointed out in [9]. From a computational viewpoint, the rectangular building approximation is more complicated than using knife edges and requires second order diffraction coefficients. These coefficients, as will be proven, perform extremely well even in the worst case scenario when both antennas are aligned with the building rooftop.

- Two-building profile. This shape represents two buildings parallel to each other that have the same height, as shown in Fig. 1. Buildings with almost the same height challenge the ray-tracing method because of the grazing condition that is created. The worst case scenario occurs when the two buildings have exactly the same height and both the transmitter and the receiver are aligned with the top of the buildings. Our experiments show that the theoretical prediction of the polygonal line simulator is in very good agreement with the measurements.

- Three-building profile. This shape represents three buildings with unequal height and shape. This profile was chosen to determine the relative importance of the contributions from different trajectories to the overall measured field.

Preliminary results of this investigation were given in [12], [13], [14].

\section{Single-Building Profile}

The single-building profile is the simplest approximation of a building obstruction along the path joining the transmitter to the receiver. Because of its rectangular shape, there are two diffracting edges at the top of the building model. From a computational viewpoint, even though this appears to be a very simple case, the field diffracted past these two edges cannot be computed using only first order diffraction coefficients, but at least second order diffraction coefficients must be applied. To prove this statement, three different configurations classified upon the position of the transmitter are examined. In all these configurations, the transmitter is kept at a constant height, while the receiver is moved vertically at small increments of a fraction of the wavelength $\lambda$. The first configuration analyzes the transmitter at a constant height above the rooftop level. The measurement results are shown in Fig. 7 and Fig. 8 for hard and soft polarization, respectively.

For both polarizations, two wide oscillations are observed in the line-of-sight (LOS) region. They are the consequence of the interference between the ray that goes directly from the transmitter to the receiver and the ray reflected on the rooftop of the building. The oscillations seen in the shadow region are due to the interference between the trajectories containing reflections from the ground with the trajectories containing only diffractions by the edges of the 


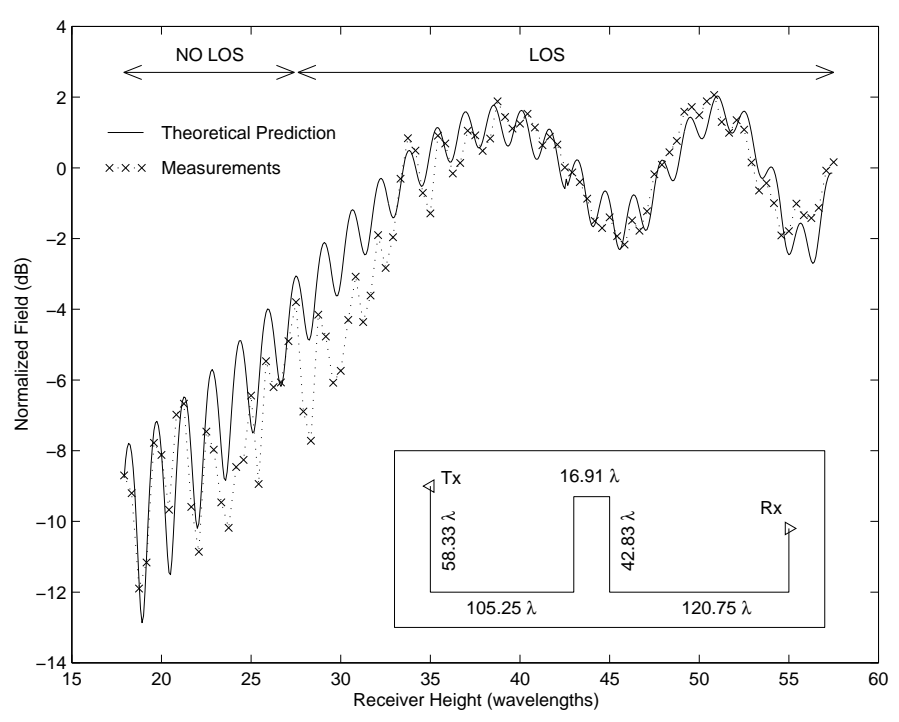

Fig. 7. Propagation with Tx above rooftop level. Hard polarization case: mean error: $0.87 \mathrm{~dB}$; standard deviation: $0.82 \mathrm{~dB}$.

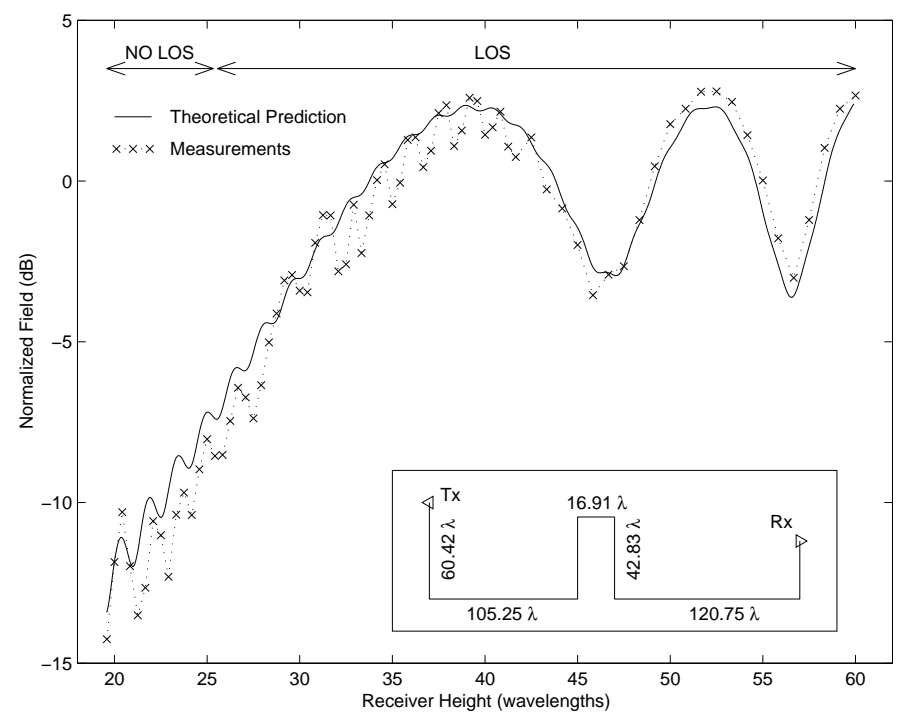

Fig. 8. Propagation with $\mathrm{Tx}$ above rooftop level. Soft polarization case: mean error: $0.79 \mathrm{~dB}$; standard deviation: $0.58 \mathrm{~dB}$.

building. The hard polarization case of Fig. 7 shows a close agreement between the measured data and the theoretical prediction with a mean error of $0.87 \mathrm{~dB}$ and a standard deviation of $0.82 \mathrm{~dB}$. The soft polarization case of Fig. 8 also proves a good agreement with a mean error of $0.79 \mathrm{~dB}$ and a standard deviation of $0.58 \mathrm{~dB}$.

The second configuration considers the transmitter below the building rooftop. The results for the hard polarization case are shown in Fig. 9. The agreement between the measurements and the theoretical prediction is given by a mean error of $1.12 \mathrm{~dB}$ and a standard deviation of $1.23 \mathrm{~dB}$. Fig. 9 also shows the theoretical prediction obtained by computing the field using only first order UTD coefficients. It is evident that, as long as the receiver is in the LOS region, this first order theoretical prediction is in close agreement with the measurements. However, when

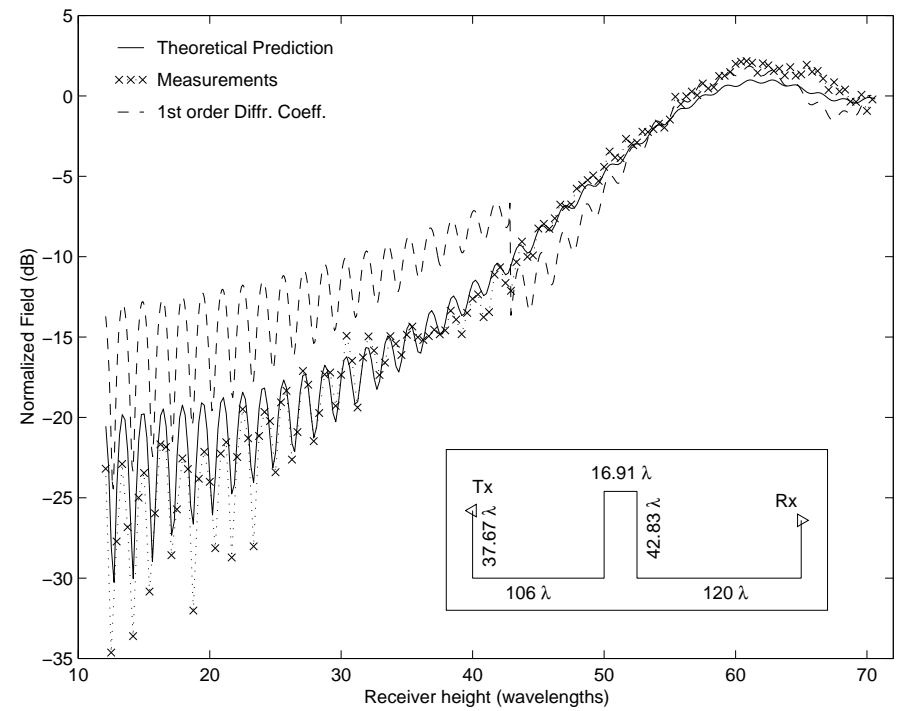

Fig. 9. Propagation with Tx below rooftop level. Hard polarization case: mean error: $1.12 \mathrm{~dB}$; standard deviation: $1.23 \mathrm{~dB}$. The computation made using only the first order UTD coefficients fails to predict the field correctly in the shadow zone.

the receiver enters the shadow region the first order theory no longer provides correct results. This proves that, even in this simple geometrical configuration, a first order UTD theory is not sufficient to provide correct results.

Reflection from the terrain deserves a special remark. When the receiving antenna approaches the ground, field oscillations up to $12 \mathrm{~dB}$ are detected for hard polarization. It is fundamental for wireless engineers to take into consideration this phenomenon when dealing with highly reflective terrain. In order to further investigate the effects of the ground plane, a particular experiment was performed. In this experiment, we reproduced the situation of Fig. 9, but without the contribution of ground reflections by covering the ground plane with absorbing material.

As shown in Fig. 10, the oscillations of the measured field are drastically reduced and a much smoother curve is obtained. The comparison with the theoretical prediction produces a mean error of $0.75 \mathrm{~dB}$ and a standard deviation of $0.56 \mathrm{~dB}$. These results show, again, a very accurate prediction achieved by the polygonal line simulator.

The results for the soft polarization case are shown in Fig. 11. Here the agreement between the experiments and the theory is expressed by a mean error of $2.90 \mathrm{~dB}$ and a standard deviation of $2.59 \mathrm{~dB}$. In this figure, the result computed using the first order UTD coefficients is also shown. It is clear that, as soon as the receiver enters the transition zone between the LOS region and the shadow region, the prediction is no longer correct. The discrepancy between theory and measurements, for lower positions of the receiver, appear to be greater than other cases previously examined. This is easily explained keeping in mind that for soft polarization the field that propagates past the building is weaker than for hard polarization. Therefore, when the normalized values of the received field are below $-30 \mathrm{~dB}$, the measurement noise starts playing an important role. 


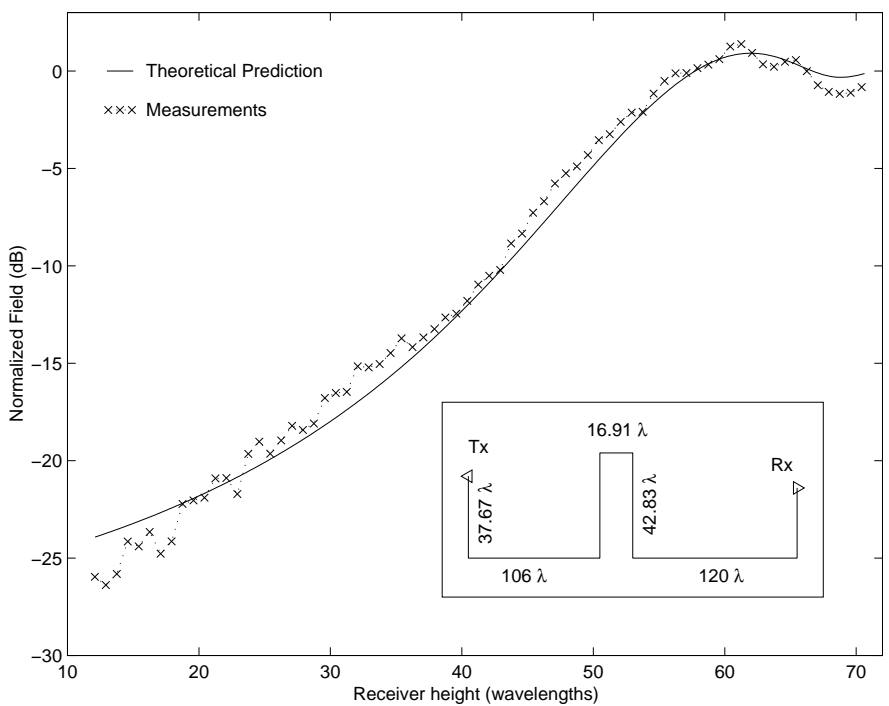

Fig. 10. Propagation with Tx below rooftop level. Hard polarization case with no ground reflection: mean error: $0.75 \mathrm{~dB}$; standard deviation: $0.56 \mathrm{~dB}$. Compare with Fig. 9 and observe that the oscillations of the measured field have practically disappeared for lower positions of the receiver.

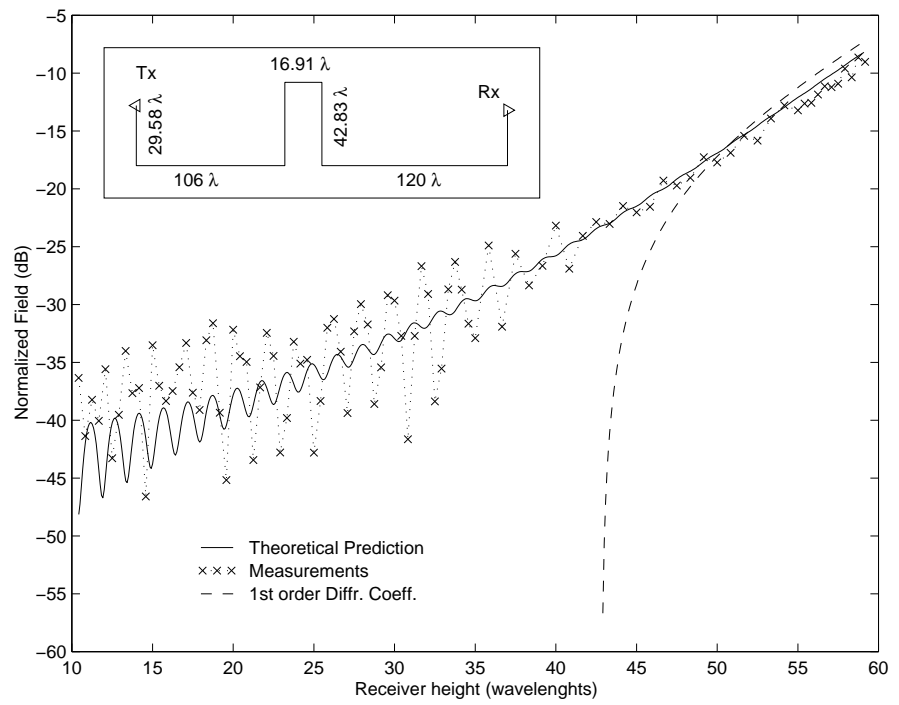

Fig. 11. Propagation with Tx below rooftop level. Soft polarization case: mean error: $2.90 \mathrm{~dB}$; standard deviation: $2.59 \mathrm{~dB}$. Notice that the field predicted using the first order UTD theory is not correct in the shadow zone.

The third configuration is the case of grazing incidence and observation aspects, as shown in Fig. 12. Here the transmitter, due to its non-negligible size, is positioned slightly below the rooftop level to guarantee that no direct ray illuminates the receiver. The receiver is moved vertically at small increments of $0.08 \lambda$. This case is challenging for the application of the ray theory because the ray approximation of the diffracted fields is not correct within the transition zones. Nevertheless, the application of the second order UTD theory provides a close agreement for both polarizations as shown in Fig. 13. In fact, for the soft polarization case, the mean error is $0.14 \mathrm{~dB}$ and the standard deviation is $0.08 \mathrm{~dB}$. In the hard polarization case,

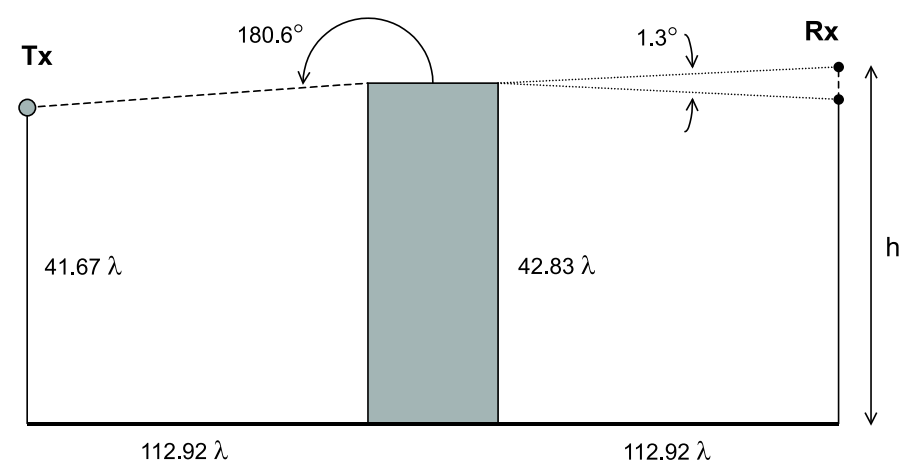

Fig. 12. Geometry for a rectangular building obstacle at almost grazing incidence and observation aspects

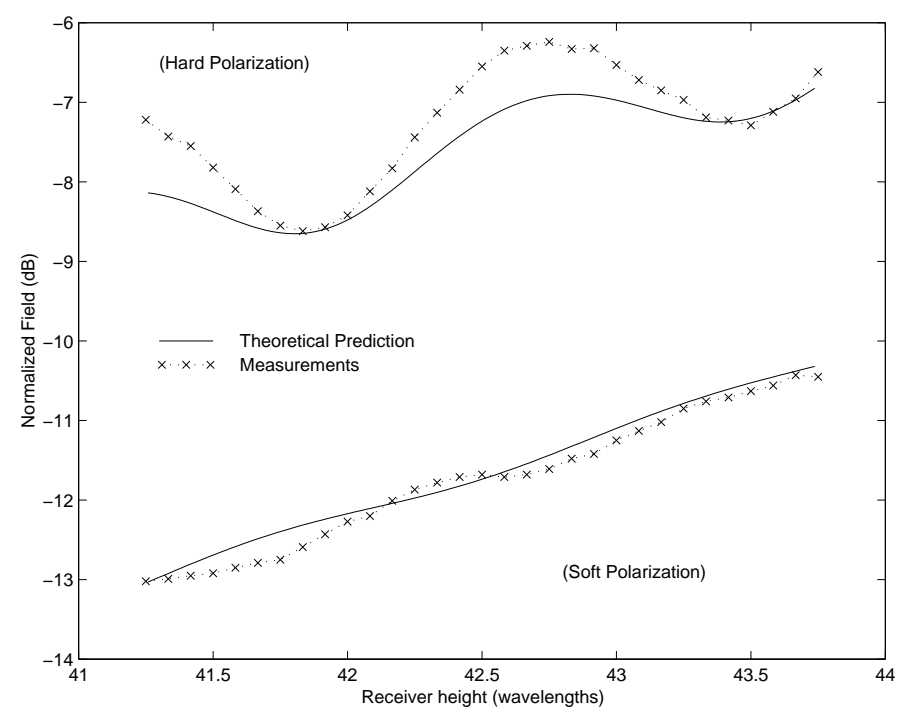

Fig. 13. Propagation at grazing incidence for the geometry shown in Fig. 12. Soft polarization case: mean error $0.14 \mathrm{~dB}$; standard deviation $0.08 \mathrm{~dB}$. Hard polarization case: mean error $0.36 \mathrm{~dB}$; standard deviation $0.27 \mathrm{~dB}$.

the mean error is $0.36 \mathrm{~dB}$ and the standard deviation is $0.27 \mathrm{~dB}$. The measurements for the single building profile show that the appropriate application of the UTD to this situation must account for a double wedge diffraction [10]. The UTD diffraction coefficients applied here are described in [7], [8] and experimentally verified in [11].

\section{Two-Building Profile}

The two-building profile represents an extension to the case of single-building obstacle analyzed in the previous section. This profile may be regarded as the simplest case of multiple rows of buildings having nearly uniform height, which is a situation of practical interest, especially in suburban areas. The case of two buildings of almost equivalent height is challenging for any ray-tracing method. Referring to the inset of Fig. 14, the challenge comes from the trajectories that illuminate the building to the left and are diffracted towards the building to the right. These trajectories are in the transition zone of the edges and, therefore, the application of the ray-tracing method must be verified. In particular, the most difficult case occurs when the trans- 


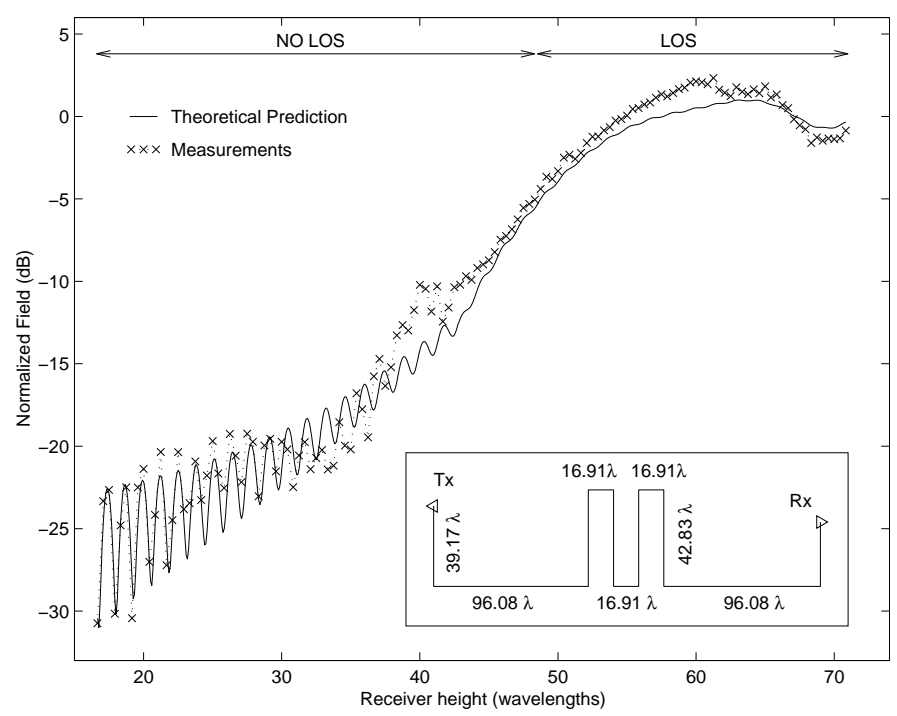

Fig. 14. Propagation with Tx below rooftops. Hard polarization case: mean error: $1.14 \mathrm{~dB}$; standard deviation: $0.85 \mathrm{~dB}$. The incidence angle is $2.18^{\circ}$.

mitter is below the building rooftop, since the field at the receiver is only due to diffraction mechanisms, which have to be carefully computed. This explains why the configurations that were measured consider the transmitter either below the rooftop or slightly below the rooftop to cause grazing incidence.

The first configuration considers the transmitter located below the level of the buildings' rooftops, while the receiver height varies from near the ground up to the line-of-sight region. The hard polarization case is examined in Fig. 14 and the comparison results in a mean error of $1.14 \mathrm{~dB}$ and a standard deviation of $0.85 \mathrm{~dB}$. The agreement is very good, but one notices stronger differences around the transition between the lit and shadow zone. This phenomenon is easy to explain on the basis that at grazing incidence the transmitter and the receiver are aligned with the four edges of the two buildings. Strictly speaking, this situation requires a diffraction coefficient of order higher than the second; however, the polygonal line simulator uses only the second order diffraction coefficients described in [7] that, nevertheless, perform very well.

To emphasize this point, we have analyzed the second configuration depicted in the inset of Fig. 15 that causes the transmitter to create grazing incidence. In fact, in Fig. 14 , the trajectory that impinges the left edge of the left building makes an angle of $2.18^{\circ}$ with the line through the building rooftops, whereas in Fig. 15 the same angle is reduced to $1.13^{\circ}$.

Fig. 15 shows the details of this comparison and corresponds to a mean error of $0.59 \mathrm{~dB}$ and a standard deviation of $0.50 \mathrm{~dB}$. Improvements could be achieved using higher order diffraction coefficients, but the benefits would be far too small when compared to the increased computational effort.

The soft polarization case is only examined for the first configuration in Fig. 16. Even though the predicted field

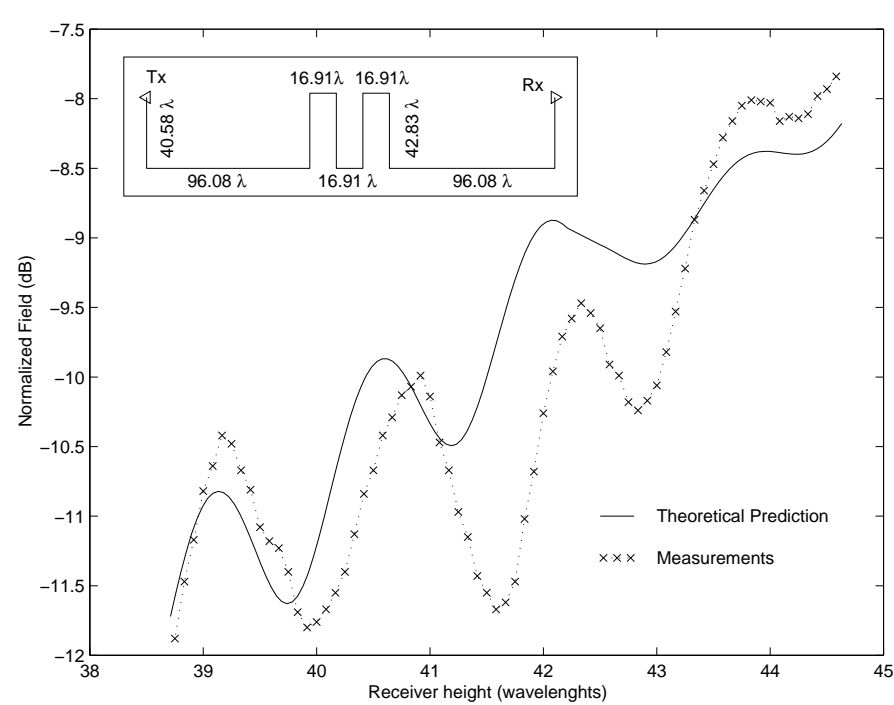

Fig. 15. Propagation with Tx below rooftops. Hard polarization case. Detail of Fig. 14 for grazing observation aspect with an incidence angle is $1.34^{\circ}$. For these data, the mean error is $0.59 \mathrm{~dB}$ and the standard deviation is $0.50 \mathrm{~dB}$

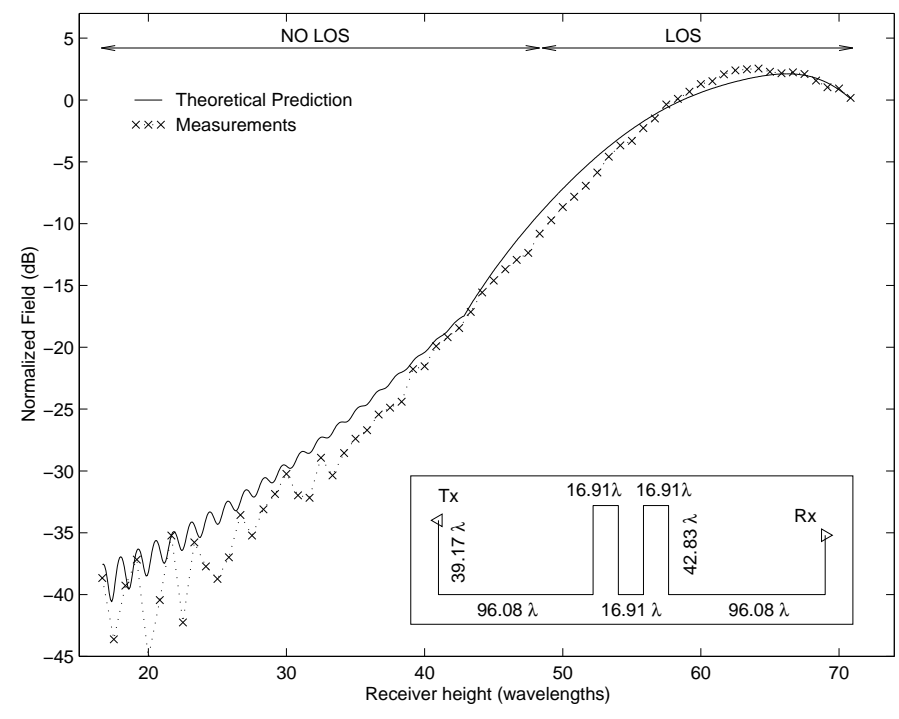

Fig. 16. Propagation with Tx below rooftops. Soft polarization case: mean error $1.53 \mathrm{~dB}$; standard deviation $1.45 \mathrm{~dB}$.

results slightly exceed the measurements in the shadow zone, the comparison still shows a very good agreement corresponding to a mean error of $1.53 \mathrm{~dB}$ and a standard deviation of $1.45 \mathrm{~dB}$. Incidentally, a similar profile has also been considered, but only theoretically, using the parabolic equation method in [15].

\section{Three-Building Profile}

The third profile contains buildings of different height and shape and is shown in Fig. 17. The purpose of this profile is to investigate propagation mechanisms more complex than those found with the previous two profiles as well as to prove that the polygonal line simulator can account for more complex situations. Referring to Fig. 18, in these experiments the transmitter was located above the rooftop 


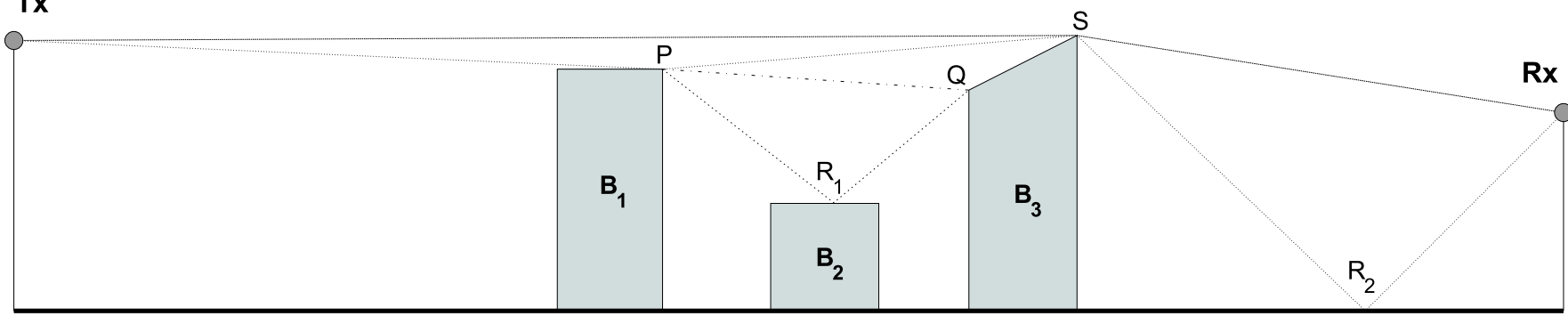

Fig. 18. Example of some trajectories that are computed by the polygonal line simulator for the profile shown in Fig. 17

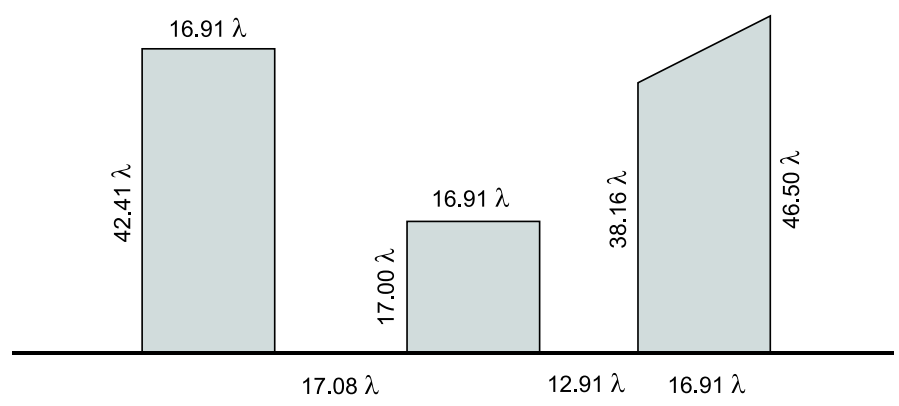

Fig. 17. Schematic of the three-building profile.

of building $B_{1}$ to allow for direct illumination of the tilted roof of building $B_{3}$. The location of $T_{x}$ also allows for an indirect illumination of the rooftop of building $B_{3}$ after a diffraction from the rooftop of building $B_{1}$. The main contributions to the received field come from the trajectories:

$$
T_{x} \rightarrow S \rightarrow R_{x}
$$

and

$$
T_{x} \rightarrow P \rightarrow S \rightarrow R_{x} .
$$

To obtain a more accurate prediction other trajectories must be included. For example, the trajectory

$$
T_{x} \rightarrow P \rightarrow Q \rightarrow S \rightarrow R_{x}
$$

may provide a non-negligible contribution especially when $T_{x}, P, Q$ are almost aligned. Furthermore, a reflection may occur on the rooftop of building $B_{2}$ and this mechanism is accounted for by the trajectory

$$
T_{x} \rightarrow P \rightarrow R_{1} \rightarrow Q \rightarrow S \rightarrow R_{x} .
$$

Incidentally, this contribution couldn't have been possible if a knife edge assumption for the shape of the buildings were made.

Moreover, when $R_{x}$ approaches the ground plane, the contribution of the trajectories that undergo a reflection at $R_{2}$ are comparable with those that propagate directly from $S$ to $R_{x}$. In fact, the evidence that these contributions are comparable is provided by the results of Fig. 19 and Fig. 20 showing oscillations for lower values of the receiver height.

By accounting for the ray contributions indicated above, as well as additional other mechanisms such as reflection

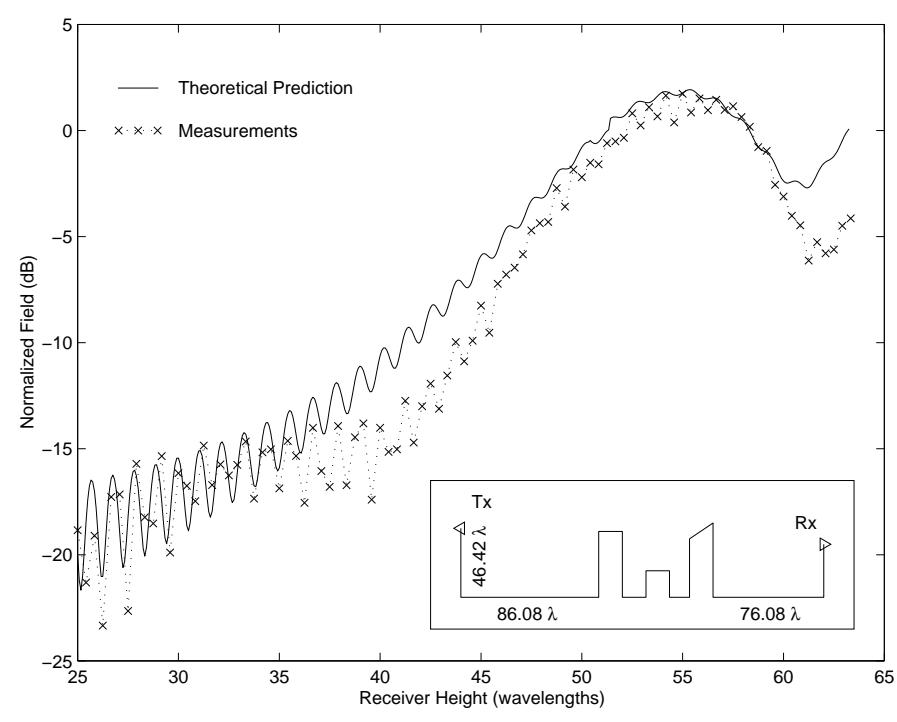

Fig. 19. Propagation with Tx above rootops. Hard polarization case: mean error $1.78 \mathrm{~dB}$; standard deviation $1.35 \mathrm{~dB}$.

from the ground plane, the theoretical predictions have been computed. The comparison for the case of hard polarization is shown in Fig. 19, and produces a mean error of $1.78 \mathrm{~dB}$ and a standard deviation of $1.35 \mathrm{~dB}$. The soft polarization case is considered in Fig. 20 and results in a mean error of $1.06 \mathrm{~dB}$ and a standard deviation of $0.85 \mathrm{~dB}$. For both cases, we can conclude that the agreement with the theoretical predictions is excellent.

\section{ViI. Conclusions}

The authors have presented experimental results conducted inside an anechoic chamber at the frequency of $25 \mathrm{GHz}$ to further verify the simulator described in [1], [2], [3]. The various comparisons show a very good agreement between theory and measurements, as proven by a mean error as low as $0.14 \mathrm{~dB}$ and a standard deviation as low as $0.08 \mathrm{~dB}$. All the measurement results are summarized in Table I and are in agreement with those published in [11]. The differences between the theory and the experiments are believed to be due to the size of the anechoic chamber used for these measurements, which limits the location of the scaled buildings to be at the very beginning of the far field zone of the antennas. Our results could be improved if the measurements were taken inside a bigger facility where the scaled buildings could be located in the far field zone of the 


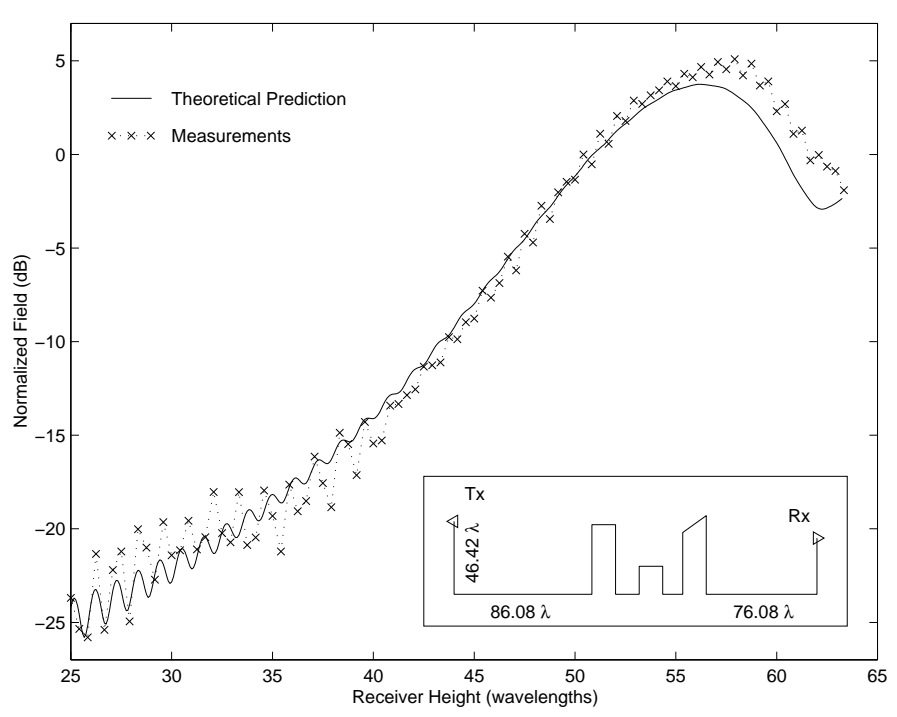

Fig. 20. Propagation with Tx above rooftops. Soft polarization case: mean error $1.06 \mathrm{~dB}$; standard deviation $0.85 \mathrm{~dB}$.

TABLE I

Summary of Measurement Statistics

\begin{tabular}{|c|c|c|c|}
\hline Configuration & Polarization & $\begin{array}{l}\text { Mean } \\
\text { Error }\end{array}$ & $\begin{array}{l}\text { Standard } \\
\text { deviation }\end{array}$ \\
\hline \multirow{3}{*}{$\begin{array}{l}\text { Single building } \\
\text { Tx-above }\end{array}$} & & & \\
\hline & hard & $0.87 \mathrm{~dB}$ & $0.82 \mathrm{~dB}$ \\
\hline & soft & $0.79 \mathrm{~dB}$ & $0.59 \mathrm{~dB}$ \\
\hline \multirow[t]{3}{*}{ Tx-below } & hard & $1.12 \mathrm{~dB}$ & $1.23 \mathrm{~dB}$ \\
\hline & hard (no refl.) & $0.75 \mathrm{~dB}$ & $0.56 \mathrm{~dB}$ \\
\hline & soft & $2.90 \mathrm{~dB}$ & $2.59 \mathrm{~dB}$ \\
\hline \multirow[t]{2}{*}{ grazing } & hard & $0.36 \mathrm{~dB}$ & $0.27 \mathrm{~dB}$ \\
\hline & soft & $0.14 \mathrm{~dB}$ & $0.08 \mathrm{~dB}$ \\
\hline \multirow[t]{3}{*}{ Two buildings } & & & \\
\hline & hard & $1.14 \mathrm{~dB}$ & $0.85 \mathrm{~dB}$ \\
\hline & soft & $1.53 \mathrm{~dB}$ & $1.45 \mathrm{~dB}$ \\
\hline \multirow[t]{3}{*}{ Three buildings } & & & \\
\hline & hard & $1.78 \mathrm{~dB}$ & $1.35 \mathrm{~dB}$ \\
\hline & soft & $1.06 \mathrm{~dB}$ & $0.85 \mathrm{~dB}$ \\
\hline
\end{tabular}

antennas. These results point out that the trajectories considered by the polygonal line simulator are capable of carefully describing the behavior of the field. In this sense, the ray tracing algorithm of the simulator is very accurate. The polygonal line simulator, however, is not limited to perfect electrical conductors, but can account for other types of material using an appropriate surface impedance value on each segment of the polygonal line that describes the geometry of the two-dimensional environment. The importance of this research is the comparison of the theoretical method with measurements inside a controlled environment. This allows for a validation of the theoretical method, without the influence of external parameters that are unavoidable in the case of open field measurements. To the best of the authors' knowledge there is only another study, reported in [16], that describes measurements on a scaled model.

A time-domain analysis is conducted in [17], and a com- parison of the two-dimensional simulator with empirical models is shown in [18].

\section{ACKNOWLEDGMENTS}

The authors are indebted to Dr. T. Monte for his technical assistance and for providing the sector antennas. The authors are thankful to the National Science Foundation which supported this research under grant ECS-9979413. The authors would like to thank the UIC Machine shop for manufacturing the scaled buildings and related items; in particular many thanks go to Mr. Art Sawczuk, Mr. Walter Schindler and Mr. Marian Balaban. Finally, they would like to thank Mr. U. G. Crovella for his assistance in the measurements, and the Reviewers for their helpful suggestions.

\section{REFERENCES}

[1] Erricolo, D. and P. L. E. Uslenghi, "Two-dimensional simulator for propagation in urban environments," IEEE Trans. Veh. Technol., Vol. 50, no.4, pp. 1158-1168, July 2001.

[2] Erricolo, D., Wireless Communications in an Urban Enviroment, Ph.D. thesis, University of Illinois at Chicago, Chicago, IL, USA, 1998.

[3] Erricolo, D., and P. L. E. Uslenghi, "Two dimensional ray tracing simulator for radiowave propagation in urban areas with arbitrary building shape and terrain profile," in National Radio Science Meeting, Atlanta, GA, USA, June 1998.

[4] Kouyoumjian, R. G., and P. H. Pathak, "A uniform geometrical theory of diffraction for an edge in a perfectly conducting surface," Proc. IEEE, vol. 62, no. 11, pp. 1448-1461, Nov. 1974.

[5] Tiberio, R. G. Manara, G. Pelosi, and R. G. Kouyoumjian, "High frequency diffraction by a double wedge," in 1985 IEEE Antennas and Propagation Society International Symposium, pp. 443-446, 1985.

[6] Herman, M. I., and J. L. Volakis, "High frequency scattering by a double impedance wedge," IEEE Trans. Antennas Propagat., vol. 36, no. 5, pp. 664-678, May 1988.

7] Albani, M., F. Capolino, S. Maci, and R. Tiberio, "Diffraction at a thick screen including corrugations on the top face," IEEE Trans. Antennas Propagat., vol. 45, no. 2, pp. 277-283, Feb. 1997.

8] Capolino, F., M. Albani, S. Maci, and R. Tiberio, "Double diffraction at a pair of coplanar skew edges," IEEE Trans. Antennas Propagat., vol. 45, no. 8, pp. 1219-1226, Aug. 1997.

[9] Erricolo, D., and P. L. E. Uslenghi, "Knife edge versus double wedge modeling of buildings for ray tracing propagation methods in urban areas," in National Radio Science Meeting, Boulder, CO, USA, Jan. 1998.

[10] Erricolo, D., "Experimental validation of second order diffraction coefficients for computation of path-loss past buildings," IEEE Trans. Electromagn. Compat., to apper in Feb. 2002 issue.

[11] Albani, M., P. Piazzesi, F. Capolino, S. Maci, and R. Tiberio, "Shielding Effect of a Thick Screen with Corrugations," IEEE Trans. Electromagn. Compat., vol. 40, no. 3, pp. 235-239, Aug. 1998.

[12] Crovella, U. G., G. D'Elia, D. Erricolo, and P. L. E. Uslenghi, "Comparison between measurements on a scaled model and a ray-tracing method for propagation in urban environments," in National Radio Science Meeting, Boulder, CO, USA, Jan. 2001.

[13] D'Elia, G., "Path loss measurements on scaled models and comparison with propagation predictions in urban environments," M.S. thesis, University of Illinois at Chicago, 2001.

[14] D'Elia, G., D. Erricolo, and P. L. E. Uslenghi, "Path loss measurements on scaled models and comparison with propagation predictions in urban environments" Proceedings of the International conference on Electromagnetics in Advanced Applications (ICEAA), Turin, Italy, Sept, 2001.

[15] Janaswamy, R., and J. Bach Andersen, "Path loss predictions in urban areas with irregular terrain topography," Wireless Personal Communications, vol. 12, pp. 255-268, 2000. 
[16] Brown, P. G., and C. C. Constantinou, "Investigations on the prediction of radiowave propagation in urban microcell environment using ray-tracing methods," IEE Proc Microw Antennas Propagat, vol. 143, no. 1, pp. 36-42, Feb. 1996.

[17] Erricolo, D., U. G. Crovella, and P.L.E. Uslenghi, "Measurements on scaled models of urban environments and comparisons with ray-tracing propagation simulation.," IEEE Trans. Antennas Propagat., this issue.

[18] Erricolo, D., and P.L.E. Uslenghi, "Comparison between raytracing approach and empirical models for propagation in urban environments.," IEEE Trans. Antennas Propagat., this issue.

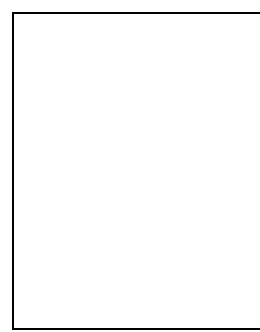

Danilo Erricolo (S'97 - M'99) received the Laurea degree of Doctor in Electronics Engineering (summa cum laude) from the Politecnico di Milano, Italy, in 1993, and the $\mathrm{Ph} . \mathrm{D}$. degree in Electrical Engineering and Computer Science from the University of Illinois at Chicago, USA, in 1998. He is a research scientist with the College of Engineering of the University of Illinois at Chicago. He serves as Senior Associate Editor for the IEEE Antennas and Wireless Propagation Letters. His current interests are on the application of electromagnetic scattering to wireless communications. Dr. Erricolo was awarded twice both the Andrew Foundation Fellowship and the Beltrami Foundation Fellowship.

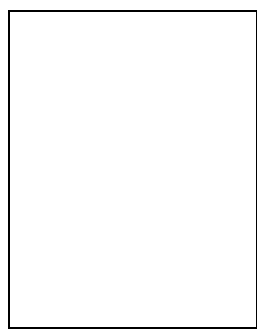

Giuseppe D'Elia (S'01) received the M.S. degree in Electrical Engineering and Computer Science from the University of Illinois at Chicago, USA, in 2001. He is studiyng towards the Laurea degree of Doctor in Electronics Engineering at the Politecnico di Torino, Italy. His research interests are in the field of electromagnetic scattering and propagation.

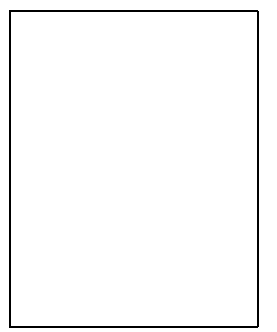

Piergiorgio L. E. Uslenghi (SM '70 - F' 90 ) was born in Turin, Italy in 1937 . He received the Doctorate in Electrical Engineering from the Polytechnic of Turin, and the M.S. and Ph.D. degrees in Physics from the University of Michigan, Ann Arbor in 1960, 1964 and 1967, respectively. He has been an Assistant Professor at the Polytechnic of Turin (1961), an Associate Research Engineer at Conductron Corporation, Ann Arbor, Michigan (1962-1963), and a Research Physicist at the Radiation Laboratory of the University of Michigan (1963-1970). In 1970, he joined the University of Illinois at Chicago, where he held a number of positions, including Founder and first Director of the Communications Laboratory (1976-1978), Founder and Director of the Electromagnetics Laboratory (1991-present), Professor of Electrical Engineering and Computer Science (1974-present), Associate Dean of the College of Engineering (1982-1987; 1994-present), and Acting Head of the Department of Electrical Engineering and Computer Science (19992000).

Dr. Uslenghi's research interests encompass antennas, microwaves, scattering theory and applications, modern optics, and applied mathematics. He has published five books and over 150 papers, is a past editor of Electromagnetics and of the IEEE Transactions on Antennas and Propagation, and has served for many years on the editorial boards of the Journal of Electromagnetic Waves and Applications, the European Journal of Telecommunications, and Alta Frequenza.

Dr. Uslenghi is a member of the Antennas and Propagation, Microwave Theory and Techniques, and Electromagnetic Compatibility Societies of IEEE. He served as Secretary-Treasurer, Vice-Chair and Chair of the Joint AP/MTT Chicago Chapter of IEEE twice, in 1975-1978 and in 1989-1992. He was the General Chair of the 1992 IEEE-APS International Symposium and URSI/NEM Meeting and the Organizer and Chair of the 1976 National Conference on Electromagnetic Scattering, both held in Chicago. He served as an elected member of the IEEE/APS Administrative Committee (1994-1996) and as Vice President (2000) and President (2001) of the IEEE Antennas and Propagation Society. He is a member of the Joint Committee on Future Symposia of IEEE/APS and USNC-URSI (1994-present), and was a member of the IEEE Heinrich Hertz Medal Committee (1992-1997). In 1990, he was elected Fellow of the IEEE for fundamental contributions to electromagnetic scattering theory and to engineering education.

Dr. Uslenghi was Chairperson of the Technical Activities Committee (1994-1996) and Vice-Chair (1997-1999) of USNC-URSI Commission B and is now Chair-Elect of Commission B (2000-2002). He is also a member of USNC-URSI Commission D. He has organized several special sessions at IEEE/APS International Symposia and URSI Meetings on such topics as nonlinear electromagnetism, scattering by wedges, advanced materials for electromagnetic applications, recent developments in scattering, and novel mathematical techniques in electromagnetics. He is the Chair of the Scientific Committee of the International Conference on Electromagnetics in Advanced Applications. He is a member of Phi Beta Kappa and Sigma Xi. 